\title{
LEVANTAMENTO ESTATÍSTICO DAS REPROVAÇÕES NO CURSO DE GRADUAÇÃO EM ENFERMAGEM DA UNIVERSIDADE ESTADUAL DE LONDRINA ${ }^{1}$
}

\author{
MITSUKO OHNISHI \\ IWA KEIKO AIDA UTYARA ${ }^{3}$ \\ MARIA INEAS DE ALMEIDA \\ LÚCIAMARIA MAISTRO \\ CECILIA EIKO FUJIWARA
}

OHNISHI, Mitsuko; UTYAMA, Iwa Keiko Aida; ALMEIDA, Maria Inês de; MAISTRO, Lúcia Maria; FUJIWARA,
Cecília Eiko. Levantamento estatístico das reprovaços no Curso de Graduaçø̃o em Enfermagem da
Universidade Estadual de Londrina. Semina: Ci. Biol.Saúde, Londrina, ‥ 16, n. 2. p. 272-276, jun. 1995.

RESUMO: Através deste estudo, os autores realizaram um levantamento estatístico de reprovaçōes por disciplinas no ciclo básico e profissionalizante do Cusso de Enfermagem, nos periodos de 1984 @ 1988, na Universidade Estadual de Londrina.

\section{PALAVRAS-CHAVE: reprovação}

\section{1 - INTRODUÇÃO}

BRASIL (1979) realizou no período de 1966 a 1972. um levantamento sobre a relação entre o número de vagas ofertadas no vestibular e o número de matriculas efetuadas nas Escolas de Enfermagem do Pais, observando que a oferta de vagas foi sempre maior que o número de matrículas iniciais.

MORRIS \& RUSSO (1979), preocupados com a elevada taxa de abandono no curso de enfermagem, efetuaram estudos com enfermeiros e estudantes de enfermagem para identificar as causas deste problema.

ARCURI et al (1983) também observaram que o índice de abandono, no período de 1977 e 1979 da Escola de Enfermagem da Universidade de São Paulo, ultrapassou os $25 \%$ dos 80 alunos matriculados. Esses autores realizaram a pesquisa no inicio do ciclo profissionalizante, na tentativa de melhor conhecer os alunos e colher dados a serem utilizados nas disciplinas subseqüentes.

Os autores vêm observando a diminuiçăo de alunos matriculados na primeira disciplina do ciclo profissionalizante, do Curso de Enfermagem e Obstetrícia da Universidade Estadual de Londrina. Sendo este um dos motivos de preocupações, enquanio integrantes de uma instituição de ensino superior, propusemo-nos a realizar o presente estudo.

Além do objetivo de verificar o índice de reprovação por disciplina dos ciclos básicos e profissionalizantes, pretende oferecer subsídios para reflexão sobre o problema, e para a realização de novos estudos em outras instítuiçes, contribuindo para o progresso do ensino de enfermagem.

\section{METODOLOGIA}

\subsection{População}

Participaram deste estudo todos os alunos matriculados no periodo de $1^{\circ}$ semestre de 1984 a $2^{\circ}$ semestre de 1988, nas disciplinas do Curso de Graduação em Enfermagem e Obstetricia da Universidade Estadual de Londrina.

\subsection{Coleta de datos}

A coleta de dados iniciou-se abril de 1989 , concluindo-se em julho de 1991, pelos próprios autores.

Realizouse a coleta de dados na Divisão de Arquivo Geral (D.A.G.), da Coordenadoria de Assuntos de Graduação (C.A.E.), Diretoria de Controle Acadêmico (D.C.A.) da Universidade Estadual de Londrina.

Encontrou-se inviabilidade de coletar dados de alunos por disciplina do ciclo básico, porque o sistema vigente de ensino é o de crédito, em que alunos de vários cursos se matriculam em disciplinas oferecidas neste ciclo. Constatou-Se, através da Assessoria de Dadose Relatorios da Coondenadoria de Assuntos Educacionais da Universidade Estadual de Londrina, que os dados referidos, na ocasing of a coletr j haviam sido

1 CCS - Departamento de Enfermagem - Universidade Estadual de Londrina, Londriva, PR, Brasi. Caixa POStal 6001, CEP 86051-970.

2 CCS - Departamento de Enfermagem - Universidede Estadual de Londina, Londrina, PR, Brasi, Caime POStal 6001, CEP 86051-970. COREN n 4.615 - PR.

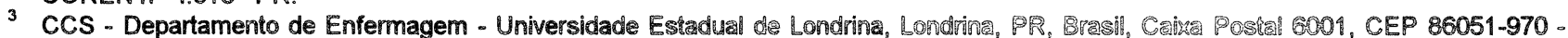
COREN n 12.868 - PR.

- Alunos do Curso de Graduaço em Enfemagem da Universidade Estadual de Londrina. 
microfilmados e que, de certa forma, o levantamento se tornaria um tanto complexo e demorado. Este fato impossibilitou a obtenção do número total de alunos de enfermagem matriculados nas disciplinas do ciclo básico.

O levantamento total dos dados, referentes ao ciclo profissionalizante, foi possivel ser realizado, pois estes encontravam-se arquivados no Centro de Ciências da Saúde da U.E.L.

\subsection{Tabulação e Análise dos Dados}

Os dados foram tabulados manualmente.

\section{RESULTADOS E DISCUSSÃO}

Os dados encontrados no presente estudo serão apresentados sob forma de tabelas, através de uma distribuição numérica.

As Tabelas 1, 2 e 3 mostram as reprovações nas disciplinas do ciclo básico; a tabela 4 trata da freqüência de reprovação nas mesmas disciplinas; e as tabela 5 e 6 demonstram o total de alunos matriculados por disciplina e número de reprovações no ciclo profissionalizante.

O percentual foi calculado considerando-se o número de matrícula oferecido pela U.E.L. para o curso de Enfermagem, (30 por semestre) e, como são analisados 10 semestres, chegou-se ao número total de alunos $n=300$, no ciclo básico.

TABELA 1 - REPROVAÇÕES DOS ALUNOS NAS DISCIPLINAS DO $1^{\circ}$. PERIOODO DO CURSO DE ENFERMAGEM, NO PERIODO DE $1^{\circ}$. SEMESTRE DE 1984 A $2^{\circ}$. SEMESTRE DE 1988, SEGUNDO DADOS DA DIVISÃO DE ARQUIVO GERAL " UEL. PR - 1989.

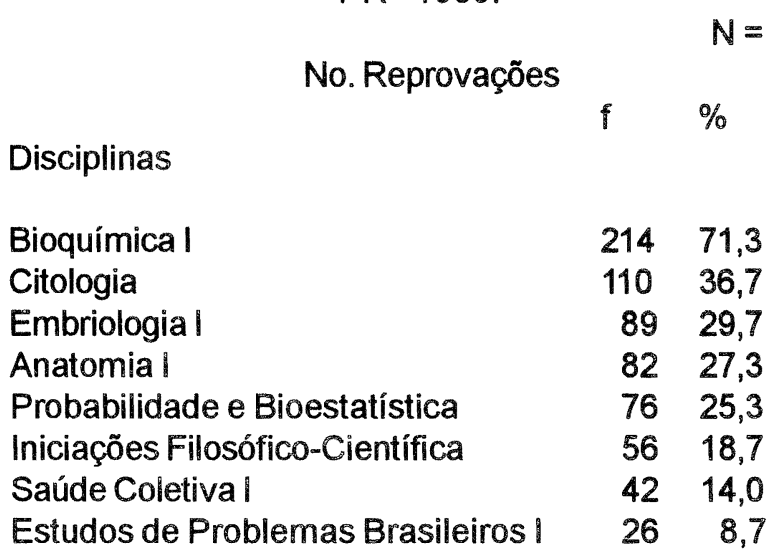

Nota-se, na Tabela 1, que o maior índice de reprovação dos alunos ocorre na disciplina de Bioquímica I $71,3 \%$; seguidos com $36,7 \%$ em Citologia; com $29,7 \%$ em Embriologia 1; com 25,3\% em Probabilidade e Bioestatística; com 18,7\% em Iniciação a FilosóficoCientífica: com 14,0\% em Saúde Coletiva I, e por último com $8.7 \%$ em Estudos de Problemas Brasileiros 1.
TABELA 2 - REPROVAÇÕES DOS ALUNOS NAS

DISCIPLINAS DO $2^{\circ}$. PERÍODO DO CURSO DE

ENFERMAGEM, NO PERIODO DE $1^{\circ}$. SEMESTRE

DE 1984 A $2^{\circ}$. SEMESTRE DE 1988 , SEGUNDO

DADOS DA DMISÃO DE ARQUIVO GERAL - UEL. PR-1989.

$$
\text { No. Reprovaçoses }
$$$$
N=300
$$

Disciplinas

Fisiologia

Histologia |

Imunologia Geral

Genéticall

Microbiologia Geral

Psicologia do Interrelacionamento

Humano

Estudo de Problemas Brasileiros 11

$\begin{array}{ll}158 & 71,3 \\ 156 & 36,7 \\ 73 & 29,7 \\ 70 & 27,3 \\ 63 & 25,3 \\ & \\ 20 & 18,7 \\ 16 & 19,0\end{array}$

Arravés da Tabela 2, observa-se que o maior número de reprovações no 20 periodo ocome com $71.3 \%$ na disciplina de Fisiologia 1 , e em seguida com $36,7 \%$ em Histologia I; com 29,7\% em Imunologia Geral; com $27,3 \%$ em Genética II; com 25,3\% em Microbiologia Geral e, por último, situam-se as disciplinas de Psicologia do Interrelacionamento Humano Estudos de Problemas Brasileiros 11 com 18,7\% e 14,0\% respectivamente.

\section{TABELA 3-REPROVACÕES DOSALUNOS NAS DISCIPLINAS DO 3. PERIODO DO CURSO DE ENFERMAGEM, NO PERIODO DE 1 . SEMESTRE DE 1984 A2. SEMESTRE DE 1988, SEGUNDO DADOS DA DMISÃO DE ARQUVO GERAL - UEL. PR $=1989$}

$$
\text { No. Reprovações }
$$$$
\mathbb{N}=300
$$

Disciplinas

$\begin{array}{lrr}\text { Processos Patológicos Gerais } & 90 & 13,3 \\ \text { Elementos de Ciências Sociais } & & \\ \text { Aplicados à Saúde } & 37 & 12,3 \\ \text { Famacologiall } & 26 & 8,7 \\ \text { Parasitologia Geral } & 17 & 5,7 \\ \text { Deontologia Médica e Enfemagem } & 14 & 4,7 \\ \text { Epidemiologia e Saneamentol } & 10 & 3,3 \\ \text { Introdução e Histónia da Enfemagem } & 1 & 1,0\end{array}$

Obserya-se, na Tabela 3, que 13,3\% de reprovacoes ocorrem na disciplina Processos Patológicos Gerais; em seguida com 12.3\% em Elementos de Ciências Sociais Aplicados à Saúde; com 8,7\% em Farmacologia II; com 5,7\% em Parasilologia Gerai; com 4,7\% em Deontologia Médica e Enfemagem; com 3,3\% em Epidemiologia e Saneamento le por untimo com $1,0 \%$ Introdução e História da Enfemagem. 
A diminuição das reprovações neste período se deve, provavelmente, ao fato que, através do alto índice de reprovações no $1^{2}$ e $2^{2}$ períodos do curso, o grupo de alunos que participava do $3^{0}$ período apresentava melhor índice de aproveitamento na aprendizagem.

TABELA 4 - FREQÜÊNCIA DE REPROVAÇÃO DOS ALUNOS NAS DISCIPLINAS DO CICLO BÁSICO CURSO DE ENFERMAGEM, NO PERÍODO DE $1^{\circ}$ SEMESTRE DE 1984 A $2^{\circ}$ SEMESTRE DE 1988, SEGUNDO DADOS DA DIVISÃO DE ARQUIVO GERAL - UEL - PR - 1989.

Freq. Reprovação

Disciplinas

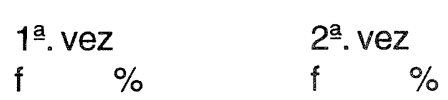

Bioquímica I

Fisiologia I

Histologia !

Citologia

Embriologia

Anatomia

Probabilidade e

Bioestatística

$\begin{array}{ll}81 & 27,0 \\ 89 & 29,7 \\ 89 & 29,7 \\ 69 & 23,0 \\ 67 & 22,8 \\ 62 & 20,7\end{array}$

6321,0

$\begin{array}{rr}86 & 28,7 \\ 54 & 18,0 \\ 61 & 20,3 \\ 32 & 10,7 \\ 16 & 5,3 \\ 15 & 5,0 \\ & \end{array}$

$10 \quad 3,3$

A tabela 4 mostra as disciplinas que tiveram mais de $25 \%$ de reprovações no ciclo básico, tendo a disciplina Bioquímica I com maior número de reprovações, 81 alunos reprovados pela primeira vez; 86 alunos pela $2^{\underline{a}}$ vez; 39 alunos pela $3^{\underline{a}}$ vez $e$ oito alunos pela $4^{\underline{a}}$ vez.

\section{TABELA 5 - ALUNOS MATRICULADOS POR DISCIPLINA DO CICLO PROFISSIONALIZANTE DO CURSO DE

\begin{tabular}{|c|c|c|c|c|c|c|c|c|c|c|c|c|}
\hline & $1 \% / 84$ & $2 \% / 84$ & $1 \% / 85$ & $2 \% / 85$ & $1 \% / 86$ & $2^{\circ} / 86$ & $1 \% / 87$ & $2 \% / 87$ & $19 / 88$ & $2^{\circ} / 88$ & Total & Médi \\
\hline \multicolumn{13}{|l|}{ Disciplinas } \\
\hline Fundamentos de Enfermagem & 32 & 22 & 15 & 31 & 14 & 17 & 11 & 17 & 24 & 20 & 203 & 20,3 \\
\hline Estágio Supervis. em Fundamentos de Enfermagem & 32 & 23 & 15 & 31 & 14 & 19 & 11 & 15 & 26 & 23 & 209 & 20,9 \\
\hline Liderança & 32 & 25 & 22 & 26 & 17 & 17 & 13 & 21 & 22 & 22 & 217 & 21,7 \\
\hline Enfermagem Médica & 20 & 31 & 24 & 15 & 29 & 13 & 17 & 12 & 16 & 24 & 201 & 20,1 \\
\hline Estágio Supervisionado em Enfermagem Médica & 20 & 31 & 24 & 15 & 29 & 15 & 19 & 13 & 17 & 26 & 209 & 20,9 \\
\hline Enfermagem Cirúrgica & 20 & 31 & 24 & 15 & 29 & 13 & 18 & 11 & 16 & 24 & 201 & 20,1 \\
\hline Estágio Superv. em Enfermagem em Centro Cirúrgico & 20 & 31 & 24 & 15 & 29 & 15 & 19 & 13 & 17 & 25 & 208 & 20,8 \\
\hline Enfermagem Ginecológica & 25 & 20 & 29 & 25 & 17 & 27 & 14 & 14 & 13 & 17 & 201 & 19,9 \\
\hline Estágio Superv. em Enfermagem Obstétrica & 26 & 21 & 29 & 25 & 17 & 27 & 14 & 14 & 12 & 16 & 201 & 20,1 \\
\hline Assistência ao Recém-Nascido & 25 & 20 & 29 & 25 & 17 & 27 & 14 & 14 & 13 & 17 & 201 & 20,1 \\
\hline Estágio Superv. em Assistência ao Recém-Nascido & 25 & 20 & 29 & 26 & 18 & 27 & 14 & 14 & 13 & 15 & 201 & 20,1 \\
\hline Enfermagem Pediátrica & 25 & 20 & 29 & 28 & 17 & 27 & 14 & 14 & 13 & 17 & 204 & 20,4 \\
\hline Estágio Superv. em Enfermagem Pediátrica & 25 & 20 & 29 & 27 & 17 & 27 & 14 & 14 & 13 & 17 & 201 & 20,1 \\
\hline Administração em Assistência de Enfermagem & 21 & 24 & 21 & 28 & 24 & 17 & 27 & 14 & 15 & 11 & 202 & 20,2 \\
\hline Estágio Superv. em Administ. em Assist. Enfermagem & 21 & 24 & 21 & 27 & 25 & 17 & 27 & 14 & 14 & 11 & 201 & 20,1 \\
\hline Enfermagem Psiquiátrica & 21 & 25 & 20 & 28 & 23 & 18 & 27 & 14 & 14 & 11 & 201 & 20,1 \\
\hline Estágio Superv. em Enfermagem Psiquiátrica & 21 & 25 & 20 & 27 & 24 & 18 & 27 & 14 & 14 & 11 & 201 & 20,1 \\
\hline Enfermagem em Saúde Pública & 21 & 25 & 20 & 28 & 23 & 18 & 26 & 13 & 14 & 11 & 199 & 19,9 \\
\hline Estágio Superv. em Saúde Pública & 21 & 25 & 20 & 27 & 24 & 18 & 26 & 13 & 14 & 12 & 200 & 20,0 \\
\hline Enfermagem em Moléstias Transmissiveis & 21 & 25 & 19 & 29 & 23 & 18 & 20 & 14 & 14 & 12 & 195 & 19,5 \\
\hline $\begin{array}{l}\text { Estágio Superv, Enferm. de Moléstias Transmissíveis } \\
\text { (25 disciplinas) }\end{array}$ & 21 & 25 & 19 & 27 & 24 & 18 & 20 & 14 & 14 & 12 & 194 & 19,4 \\
\hline TOTAL & 586 & 616 & 590 & 605 & 546 & 493 & 455 & 352 & 387 & 433 & 5063 & \\
\hline
\end{tabular} ENFERMAGEM, DO PERÍODO DE 1984 A 1988 - UEL - LONDRINA}

$\mathrm{Na}$ tabela 5, são demonstrados os totais de alunos matriculados nas disciplinas do ciclo profissionalizante do curso de Graduação em Enfermagem da Universidade Estadual de Londrina, no período de 1984 a 1988.
Observa-se a diminuição de alunos matriculados a cada ano, principalmente no $2^{\circ}$ semestre de 1987 que apresentou o menor índice, com um total de 352 alunos, quando se esperava o total de 750 alunos, sem reprovação. 
TABELA 6 - ALUNOS MATRICULADOS E REPROVADOS DAS DISCIPLINAS DO CICLO PROFISSIONALIZANTE DO CURSO DE ENFERMAGEM, NO PERÍODO DE $1^{\circ}$ SEMESTRE DE 1984 À $2^{\circ}$ SEMESTRE DE 1988 , SEGUNDO DADOS DA DIVISÃO DE ARQUIVO GERAL - UEL - PR - 1989.

Total de alunos matriculados e reprovados

Disciplinas

Fundamentos de Enfermagem

Estágio Supervis. em Fundamentos de Enfermagem

Liderança em Enfermagem

Enfermagem Médica

Estágio Supervisionado em Enfermagem Médica

Enfermagem Cirúrgica

Estágio Superv. em Enfermagem Cirúrgica

Enfermagem em Centro Cirúrgico

Estágio Superv. em Enfermagem em Centro Cirúrgico

Enfermagem Ginecológica

Estágio Superv. em Enfermagem Ginecológica

Enfermagern Obstétrica

Estágio Superv. em Enfermagem Obstétrica

Assistência ao Recém-Nascido

Estágio Superv. em Asssistência ao Recém-Nascido

Enfermagem Pediátrica

Estágio Superv. em Enfermagem Pediátrica

Administraçăo em Assistência de Enfermagem

Estágio Superv. em Administ. em Assist. Enfermagem

Enfermagem Psiquiátrica

Estágio Superv. em Enfermagem Psiquiátrica

Enfermagem em Saúde Pública

Estágio Superv. em Saúde Pública

Enfermagem em Moléstias Transmissiveis

Estágio Superv. Enferm. de Moléstias Transmissiveis

$\mathrm{Na}$ tabela 6, nota-se que, no ciclo profissionalizante, as disciplinas que mais reprovaram foram as de Fundamentos de Enfermagem com 5,4\% e Estágio Supervisionado em Fundamentos de Enfermagem com 9,1\%; em seguida a disciplina de Estágio Supervisionado em Enfermagem Cirúrgica com 4,8\%; as disciplinas Liderança em Enfermagem, Estágio Supervisionado em Enfermagem Médica, Estágio Supervisionado em Enfermagem em Centro Cirúrgico com $3,2 \%, 3,3 \%, 3,4 \%$ respectivamente.

Observa-se, então, maior número de reprovações no ciclo profissionalizante nas disciplinas Fundamentos de Enfermagem e Estágio Supervisionado em Fundamentos de Enfermagem, provavelmente por ser a primeira experiência do aluno com o ambiente hospitalar, e onde deve desenvolver as atividades de Enfermagem.

\section{CONCLUSÃO}

Através deste estudo, nota-se que os acadêmicos

$\begin{array}{lll}203 & 11 & 5,4 \\ 209 & 19 & 9,1 \\ 217 & 07 & 3,2 \\ 201 & 01 & 0,5 \\ 209 & 07 & 3,3 \\ 201 & 04 & 2,0 \\ 208 & 10 & 4,8 \\ 205 & 02 & 1,0 \\ 206 & 07 & 3,4 \\ 201 & 01 & 0,5 \\ 199 & 04 & 2,0 \\ 203 & 02 & 1,0 \\ 201 & 04 & 2,0 \\ 201 & 03 & 1,5 \\ 201 & 03 & 1,5 \\ 204 & 02 & 1,0 \\ 201 & 02 & 1,0 \\ 202 & 01 & 0,5 \\ 201 & 00 & 0,0 \\ 201 & 00 & 0,0 \\ 201 & 00 & 0,0 \\ 199 & 00 & 0,0 \\ 200 & 01 & 0,5 \\ 195 & 01 & 0,5 \\ 194 & 00 & 0,0\end{array}$

de enfermagem são reprovados com mais freqüencia nas seguintes disciplinas do ciclo básico: Bioquímica com $71,3 \%$, Fisiologia I com $52,8 \%$ e Histologia com $52,0 \%$.

Nas disciplinas do ciclo profissionalizante, os alunos foram reprovados em: Estágio Supervisionado em Fundamentos de Enfermagem com 9,1\% e Fundamentos de Enfermagem com $5,4 \%$.

A reprovação é maior no ciclo básico do que no ciclo profissionalizante.

Esses dados confirmam os de DE SORDI et al (1989) que demonstram que houve uma gradativa deterioração no rendimento do aluno que ingressa na Universidade, e que tal fato tem a ver com a falência do sistema de ensino de $1^{\circ}$ e $2^{\circ}$ graus que não garante os pré-requisitos mínimos necessários para o aluno freqüentar a Universidade.

Ressalta-se, ainda, a necessidade perante tais dados, de se dar prosseguimento a este estudo no intuito de aprofundar e analisar as possiveis causas que geraram essa realidade. 
OHNISHI, Mitsuko; UTYAMA, Iwa Keiko Aida; ALMEIDA, Maria Inês de; MAISTRO, Lúcia Maria; FUJIWARA, Cecilia Eiko. Statistics on the Failure in the Nursing Graduation Course at Londrina State University. Semina: Ci. Biol./Saíde, Londrina, v. 16, n. 2, p. 272-276, Jun. 1995.

ABSTRAT: BY means of this research, the authors show a statistic investigation of failures by subjects on the basic and professionalizing cicles of Nursing Graduate Course, from 1984 to 1988, at Universidade Estadual de Londrina.

KEYUMORDS: FailuBC

\section{REFERENNCIAS BIBLIOGRÁFICAS}

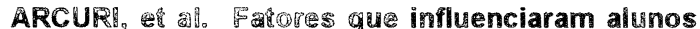
ingressantes 1981, ที

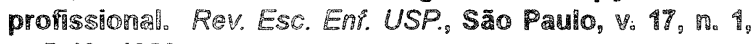
p. 5-19, 1983.

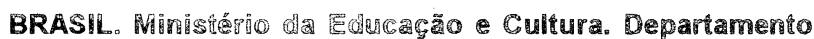

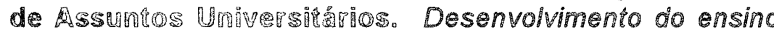
superior de enfemagem no Brasil. \$. 1 : Brasilipar. 1979.. $118 \%$
OE SOROI, M. R. Aêt a alteraço do penil do aluno ingressante ne faculdade de enfemagem da PUC CAMP seu impacto no ciclo básico. lip: Congresso Brasileiro de Enfermagem, 1989, Florianónolis. Anais plorianópolis, 1988, 15 p.

MORRIS, B. RUSSON, B. Motives of begining students for choosing mursing school. J. Nurs. Ed. Thorofare, V. 8 